\title{
HIV DNA Reservoir Increases Risk for Cognitive Disorders in CART-Naïve Patients
}

\author{
Victor G. Valcour ${ }^{1,2 *}$, Jintanat Ananworanich ${ }^{3,4,5,6}$, Melissa Agsalda ${ }^{6}$, Napapon Sailasuta ${ }^{7}$, \\ Thep Chalermchai ${ }^{3}$, Alexandra Schuetz ${ }^{8}$, Cecilia Shikuma ${ }^{6}$, Chin-Yuan Liang ${ }^{6}$, Supunee Jirajariyavej ${ }^{9}$, \\ Pasiri Sithinamsuwan ${ }^{10}$, Somporn Tipsuk ${ }^{3}$, David B. Clifford ${ }^{11}$, Robert Paul ${ }^{12}$, James L. K. Fletcher ${ }^{3}$, \\ Mary A. Marovich ${ }^{13,14}$, Bonnie M. Slike ${ }^{14,15}$, Victor DeGruttola ${ }^{16}$, Bruce Shiramizu ${ }^{6}$ for the SEARCH 011 \\ Protocol Team
}

1 Memory and Aging Center, Department of Neurology, University of California San Francisco, San Francisco, California, United States of America, 2 Division of Geriatric Medicine, Department of Medicine, University of California San Francisco, San Francisco, California, United States of America, 3 SEARCH, Thai Red Cross AIDS Research Centre, Bangkok, Thailand, 4 HIV-NAT, Thai Red Cross AIDS Research Centre, Bangkok, Thailand, 5 Faculty of Medicine, Chulalongkorn University, Bangkok, Thailand, 6 Hawaii Center for AIDS, University of Hawaii, Honolulu, Hawaii, United States of America, 7 Huntington Medical Research Institute, Pasadena, California, United States of America, 8 Department of Retrovirology, Armed Forces Research Institute of Medical Sciences, Bangkok, Thailand, 9 Taksin Hospital, Bangkok, Thailand, 10 Division of Neurology, Department of Medicine, Phramongkutklao Hospital, Bangkok, Thailand, 11 Department of Neurology, Washington University, St. Louis, Missouri, United States of America, 12 Department of Psychology, University of Missouri, St. Louis, Missouri, United States of America, 13 Division of AIDS, National Institute of Allergy and Infection Diseases of the National Institutes of Health, Bethesda, Maryland, United States of America, 14 Department of Biostatistics, Harvard School of Public Health, Boston, Massachusetts, United States of America, 15 Queen Savang Vadhana Memorial Hospital, Si Racha, Thailand, 16 Thai Red Cross AIDS Research Centre, Bangkok, Thailand

\begin{abstract}
Objectives: Cognitive impairment remains frequent in HIV, despite combination antiretroviral therapy (cART). Leading theories implicate peripheral monocyte HIV DNA reservoirs as a mechanism for spread of the virus to the brain. These reservoirs remain present despite CART. The objective of this study was to determine if the level of HIV DNA in CD14 enriched monocytes predicted cognitive impairment and brain injury.

Methods: We enrolled 61 cART-naïve HIV-infected Thais in a prospective study and measured HIV DNA in CD14 ${ }^{+}$enriched monocyte samples in a blinded fashion. We determined HAND diagnoses by consensus panel and all participants underwent magnetic resonance spectroscopy (MRS) to measure markers of brain injury. Immune activation was measured via cytokines in cerebrospinal fluid (CSF).

Results: The mean (SD) age was 35 (6.9) years, CD4 T-lymphocyte count was 236 (139) and $\log _{10}$ plasma HIV RNA was 4.8 (0.73). Twenty-eight of 61 met HAND criteria. The $\log _{10}$ CD14 $4^{+}$HIV DNA was associated with HAND in unadjusted and adjusted models $(p=0.001)$. There was a 14.5 increased odds ratio for HAND per 1 log-value of HIV DNA (10-fold increase in copy number). Plasma CD14 ${ }^{+}$HIV DNA was associated with plasma and CSF neopterin $(p=0.023)$ and with MRS markers of neuronal injury (lower $\mathrm{N}$-acetyl aspartate) and glial dysfunction (higher myoinositol) in multiple brain regions.

Interpretation: Reservoir burden of HIV DNA in monocyte-enriched (CD14 ${ }^{+}$) peripheral blood cells increases risk for HAND in treatment-naïve HIV+ subjects and is directly associated with CSF immune activation and both brain injury and glial dysfunction by MRS.
\end{abstract}

Citation: Valcour VG, Ananworanich J, Agsalda M, Sailasuta N, Chalermchai T, et al. (2013) HIV DNA Reservoir Increases Risk for Cognitive Disorders in cART-Naïve Patients. PLoS ONE 8(7): e70164. doi:10.1371/journal.pone.0070164

Editor: Stephen D. Ginsberg, Nathan Kline Institute and New York University School of Medicine, United States of America

Received March 18, 2013; Accepted June 15, 2013; Published July 31, 2013

Copyright: (c) 2013 Valcour et al. This is an open-access article distributed under the terms of the Creative Commons Attribution License, which permits unrestricted use, distribution, and reproduction in any medium, provided the original author and source are credited.

Funding: This work was supported by R01-NS053359 (NINDS/NIH - SRK), R01-NS061696 (NINDS/NIH - VV), R21-MH072388 (NIMH/NIH - VV), R01-NS053345 (NINDS/NIH - BS), and MD007584 (NIMHD/NIH - BS, CS). Added statistical assistance was supported by the National Center for Advancing Translational Sciences, National Institutes of Health (NIH), through UCSF-CTSI Grant Number UL1 TR000004. Its contents are solely the responsibility of the authors and do not necessarily represent the official views of the $\mathrm{NIH}$. This work was supported in part by a cooperative agreement (W81XWH-11-2-0174) between the Henry M. Jackson Foundation for the Advancement of Military Medicine, Inc, and the U.S. Department of Defense. The views expressed are those of the authors and should not be construed to represent the positions of the US Army or the Department of Defense. The funders had no role in study design, data collection and analysis, decision to publish, or preparation of the manuscript.

Competing Interests: The authors have declared that no competing interests exist.

* E-mail: vvalcour@memory.ucsf.edu

- Membership of the SEARCH 011 Protocol Team is provided in the Acknowledgments.

\section{Introduction}

Combination antiretroviral therapy (cART) suppresses plasma HIV viral RNA to undetectable levels for most patients but fails to universally eliminate reservoirs of HIV DNA. [1] The inability to clear these reservoirs has emerged as the Achilles heel of HIV eradication because withdrawal from treatment allows for rapid new viral replication from these sources. Although sometimes 
assumed to be quiescent, the magnitude of the reservoir in peripheral blood mononuclear cells (PBMC) has been linked to HIV disease progression and mortality. [2,3] We hypothesize that reservoirs within circulating monocytes contribute to cognitive impairment and are likely to underlie continued brain injury.

Current neurological research has focused on the brain as a protected site for HIV since not all antiretroviral medications have shown high degrees of central nervous system (CNS) penetration effectiveness (CPE). Our past work focused on the burden of HIV DNA in PBMCs, and particularly those enriched for monocytes as determined by expression of the CD14 cell surface marker. This CD $14^{+}$HIV DNA reservoir is proportionally small compared to that found in $\mathrm{CD}^{+}{ }^{+}$T-lymphocytes; however, the overall CD $14^{+}$ reservoir size has been linked to cognitive disorders among both cART-treated and untreated patients in past cross-sectional posthoc correlative studies. [4,5,6,7] A recent study linked cART regimens with higher effectiveness in monocytes to better overall cognitive performance, a finding that was independent of CPE. [8] Autopsy reports identify a monocyte/macrophage foundation to cognitive impairment even among cART treated subjects, and researchers have shown an association between soluble CD14 and lysosomal proteins secreted by macrophages to cognitive impairment and brain atrophy. $[9,10,11,12,13]$ We now test the hypothesis that $\mathrm{CD} 14^{+}$HIV DNA can identify subjects with HAND prospectively in a blinded fashion, and evaluate the mechanistic link to CNS injury by brain MRS and evaluation of CSF immune activation.

\section{Methods}

\section{Ethics Statement}

All subjects provided written informed consent. The UCSF Human Research Protection Program Committee on Human Research provided approval of the consent form and study via the Notification of Expedited Review Approval, expiry of 17 June 2013. The Institutional Review Board of the Faculty of Medicine, Chulalongkorn University provided approval of the consent form and study, stating "The Institutional Review Board of the Faculty of Medicine, Chulalongkorn University, Bangkok, Thailand, has approved the following study which is to be carried out in compliance with the International guidelines for human research protection as Declaration of Helsinki, the Belmont report, CIOMS Guideline, and International Conference on Harmonization in Good Clinical Practice (ICH-GCP)," expiry of 02 September 2013.

\section{Patient Selection}

SEARCH 011 (NCT00782808) was enrolled prospectively with referrals from community clinicians for subjects that met Thai Ministry of Public Health criteria for treatment initiation $\left(\mathrm{CD} 4^{+}\right.$ T-lymphocyte count $<350$ cells $/ \mathrm{mm}^{3}$ or symptomatic disease). [14] All were screened for PBMC HIV DNA levels to ensure a full range of HIV DNA in the final sample. Using a central randomization center where clinical staff were blinded to these levels, we aimed to enroll 30 cases with greater than and 30 cases with less than 1000 copies of HIV DNA per $10^{6}$ PBMCs. We used PBMC rather than $\mathrm{CD}_{1}{ }^{+}$cellular HIV DNA for screening because logistical challenges precluded $\mathrm{CD} 14^{+}$cell separation in real time, and because the two measures were highly correlated in our preliminary studies. We further stratified by age of greater or less than 35 years to minimize clustering by age, a factor that could impact cognition.

Clinicians remained blinded to all HIV DNA levels and laboratory technicians were blinded to clinical data, including cognitive information, for the duration of the study. Subjects were excluded for head injury, current illicit drug use or a positive urine toxicology test at either the screening or entry visit, acute concurrent illness, pre-existing neurologic or psychiatric conditions, or learning disability. We enrolled 63 subjects, but excluded two at entry when opportunistic CNS infections were discovered (toxoplasmosis and tuberculosis).

\section{Cognitive Characterization}

Trained nurse-psychometrists administered neuropsychological tests from a battery developed for international use by the World Health Organization (WHO) and modified slightly for feasibility, as previously described. $[15,16]$ The battery includes the WHO Auditory Verbal Learning Task (WHO AVLT) for learning efficiency, immediate and delayed recall; the Brief Visual Memory Task-Revised (BVMT-R); Color Trails 1 and 2; Escala de Inteligencia de Wechsler para Adultos (EIWA) Digit Symbol and Block Design Tasks; the Grooved Pegboard for both hands; Finger Tapping for both hands; Timed Gait; two verbal fluency tasks (first name and animals); and the Trail Making Test A. The study neurologist conducted a standardized examination developed by the AIDS Clinical Trials Group (ACTG). Nurses and physicians independently interviewed subjects and, when possible, interviewed proxy informants to identify functional limitations due to cognitive impairment. Individual neuropsychological test raw scores were compared to age- and education-stratified Thai normative data to generate standardized z-scores ((subject score normative score)/normative standard deviation) and were combined to provide a global composite $\mathrm{z}$-score (NPZglobal) as the arithmetic mean of all standardized scores per subject. [17].

Clinical diagnoses of cognitive impairment were assigned in a consensus conference that included the principle investigator $(\mathrm{VV})$, a U.S. HIV neurologist (DBC), and a U.S. HIV neuropsychologist (RP) using the 2007 ("Frascati") diagnostic criteria as a guide. [18] Given the limitations of the brief one-hour neuropsychological battery, clinical acumen was required to judge whether the abnormalities were mild or moderate in nature and to conclude if there was sufficient evidence for cognitive abnormalities beyond normal test variation. We allowed for slight departures from the 2007 nosology when consensus was achieved to define the following: cognitively normal (NL): testing performance deemed to be within expectations for age and educational attainment; asymptomatic neurocognitive impairment (ANI): performance deemed to be worse than expected with normal test variation (typically involving at least two domains) but without evidence of functional impairment; mild neurocognitive disorder (MND): moderately abnormal performance (typically 1 to $2 \mathrm{SD}$ below normative data) in two cognitive domains and with evidence of functional impairment; and HIV-associated dementia (HAD): severe impairment (typically worse than $-2 \mathrm{SD}$ ) in two cognitive domains with clear evidence of functional impairment. [18] Consensus conference members were blinded to HIV DNA levels.

Standard laboratory evaluations included a complete blood cell count with T-lymphocyte subsets, HIV RNA, liver profiles, blood chemistries, syphilis serology, vitamin $\mathrm{B}_{12}$ level, thyroid function tests, and hepatitis serology. Lumbar puncture was optional and obtained on 43 subjects. CSF and plasma viral loads were measured using the Amplicor HIV-1 Monitor Assay (Roche Molecular System, Inc., Branchburg, NJ).

\section{Cell Separation}

PBMCs were isolated using Ficoll Histopaque ${ }^{\circledR}$ (Sigma, St Louis MO) density gradient centrifugation and washed three times with RPMI1640 culture media (Life Technologies, Grand Island NY) 
containing 2\% heat-inactivated fetal bovine serum (FBS) (Gibco) and $1 \%$ Pen/Strep (Gibco). Monocytes were purified by magnetic bead positive selection (MiltenyiBiotec, City ST). In brief, PBMCs were incubated with anti-CD14 magnetic MicroBeads for 15 minutes on ice, washed to remove excessive beads, then loaded onto a MACS column and placed in a magnetic field. CD14 ${ }^{+}$ enriched cells were collected using the appropriate buffer provided by the manufacturer. The median purity of the $\mathrm{CD} 14^{+}$cells was 91.9\% (min: 76.9\%; max: 98.7\%) by multi-parameter flow cytometry on every fifth sample for the first 42 cases. Cells were frozen in $10 \%$ DMSO and shipped in batches to the U.S. for the HIV DNA quantification.

\section{HIV DNA Quantification}

We quantified HIV DNA using the QIAamp DNA Micro Extraction kit (Qiagen, Valencia, CA) using the ND-1000 spectrophotometer (NanoDrop Technologies; Wilmington, DE) as previously described. [19] Briefly, we used multiplex real-time PCR with HIV gag and $\beta$-globin primer pairs to amplify respective regions with VIC-labeled HIV gag and FAM-labeled $\beta$-globin probes. Using standard reference plasmids with one copy of the $\beta$ globin housekeeping gene and one copy of the HIV gag gene and appropriate positive/negative controls, samples were run in triplicate on StepOnePlus Real-Time PGR System and analyzed using the SDS 2.3 software (Applied Biosystems, Foster City, CA). The copy numbers of each sample gene were analyzed against the standard curves to determine HIV DNA copy number per $10^{6}$ cells.

\section{Plasma and CSF Cytokines}

MCP-1 and IL-6 were quantified in triplicate as part of a custom multiplex ELISA array according to the manufacturer's protocol (Quansys Biosciences, Logan UT). Data were captured on the Odyssey infrared imaging system (Li-Cor Biosciences, Lincoln, NE) and analyzed using Quansys Q-view Plus software (Quansys Biosciences). Single-analyte ELISA was performed in duplicate to detect levels of neopterin (GenWay Biotech, San Diego CA) and analyzed using SoftMax Pro (Molecular Devices, Sunnyvale CA).

\section{Brain MRS}

Subjects underwent axial 3D T1-weighted spoiled gradient echo MRI $\left(\mathrm{TE}=7 \mathrm{~ms}\right.$, TR $=11.2 \mathrm{~ms}$, flip angle $=25^{\circ}, 1 \mathrm{~mm}$ resolution) on the same GE Signa HDx 1.5T scanner (GE Healthcare, software v12-M4) with 8-channel head coil and a standard body coil. Single voxel MRS was acquired by double spin echo data acquisition (PROBE-P, TE $=35 \mathrm{~ms}$, TR $=1.5 \mathrm{~s}$ ) at four locations: left frontal white matter (FWM, 8cc), midline frontal grey matter (FGM, 8cc), occipital grey matter (OGM, 8cc), and basal ganglia (BG, 8cc) (Figure 1). Sixteen unsuppressed water free induction decays (FIDs) and 128 water suppressed FIDs were acquired for all locations, with 192 water suppressed FIDs acquired at BG. We measured $\mathrm{N}$-acetyl aspartate (NAA), choline (Cho), myoinositol (MI), glutamate+glutamine $(\mathrm{Glx})$, and creatine $(\mathrm{Gr})$. To ensure scanner stability, short echo-time (TE $=35 \mathrm{~ms}$ ) single voxel MRS was obtained using a standard spectroscopy phantom (GE Healthcare) after each scan. [20].

Data were securely transferred and processed by one author (NS) using the time domain linear combination fitting software, LCModel (version 6.2, http://s-provencher.com/pages/lcmodel. shtml). Time domain MRS data from each of the 8-channel phased array head coils were combined using unsuppressed water FIDs from each coil as scaling factor. [21] The FIDs were processed without spectral line broadening for fitting. Fittings were performed between $4.0-0.5 \mathrm{ppm}$, using a reference basis set acquired using the same data acquisition. All reference solutions were adjusted to $\mathrm{pH} 7.2$ with $0.1 \mathrm{M} \mathrm{NaOH}$. Metabolite quantification for NAA, Cr, Cho, MI, and Glx was included only if the signal to noise ratio was $>4$ and the percent standard deviations were $<20 \%$. [22].

\section{Statistical Analysis}

We used Kruskal-Wallis and student t-tests to compare HAND and non-HAND groups, and logistic regression to examine the association between HAND and clinical variables. We constructed a receiver operator characteristics (ROG) curve to determine the optimal CD $14^{+}$HIV DNA cutoff for detecting HAND, and evaluated the performance of the classifier using the area under the curve. Multiple regression models were used to relate predictors to the NPZglobal score. We also evaluated the association between HIV DNA and the three cytokines of interest (MCP-1, neopterin, and IL-6). All values were log transformed prior to inclusion in our models. Predictors included $\log _{10}$ transformed HIV DNA copy number, plasma HIV RNA, and cytokine measures. For MRS analyses, we hypothesized finding higher MI and lower NAA associated with HIV DNA, but we also examined Cho and Glx. Each voxel was analyzed independently by regressing HIV DNA copy number on each metabolite separately with age, gender, and creatine included in the models.

\section{Results}

All subjects were enrolled between March 2009 and December 2011. Among these, 35 (57\%) were female and the mean (SD) age was 34.7 (6.9) years. At consensus conference, 28 subjects met criteria for HAND: 14 with ANI, 8 with MND, and 6 with HAD. The HAND and non-HAND groups did not differ in main demographic and clinical variables (Table $\mathbf{1}$ ).

\section{HIV DNA and Cognition}

The median (IQR) HIV DNA copy number per $10^{6} \mathrm{CD} 14^{+}$ cells was $27(18,54), 68$ (46, 158), $109(66,213)$, and $138(114,265)$ for NL, ANI, MND and HAD, respectively $(p<0.001$, Figure 2). The CD14 ${ }^{+}$HIV DNA reservoir burden was associated with HAND (ANI+MND+HAD) in both univariate $(\mathrm{OR}=14.9$, $p<0.001)$ and multiple logistic models $(\mathrm{OR}=14.5, p=0.001)$ adjustment for concurrent $\mathrm{CD} 4^{+} \mathrm{T}$-lymphocyte count and plasma HIV RNA (Table 2). For given CD4 $4^{+}$T-lymphocyte and HIV RNA levels, the odds of observing HAND increased by $14.5(95 \%$ CI 3.00-69.7) per one log-value increase in HIV DNA copy number (10-fold increase in raw scale). We identified a moderate discriminative power as a diagnostic test for HAND $(\mathrm{AUC}=0.79$, Figure 3). When an optimal point, determined by Youden's index, of 45 copies of HIV DNA per $10^{6} \mathrm{CD} 14^{+}$cells is used, this model provided a sensitivity of $86 \%$ and a specificity of $70 \%$. In contrast, no correlation with cognition was observed for HIV DNA measured from the full PBMC pool prior to enriching for $\mathrm{CD}_{14}{ }^{+}$cells. The median PBMC HIV DNA (IQR) was 943 (417,2613), 1677 (243,2458), 1110 (422,4539), and 1037 $(435,2673)$ for NL, ANI, MND and HAD, respectively $(p=0.99)$ and 943 vs. 1163 copies per $10^{6}$ cells, for non-HAND vs. HAND, respectively $(p=0.87)$. CD $14^{+} \mathrm{HIV}$ DNA was not associated with plasma HIV RNA.

The association between CD14 ${ }^{+}$HIV DNA and consensus diagnosis was similarly reflected in the summary neuropsychological testing score. Simple linear regression models revealed that both HIV DNA $\left(r^{2}=0.064, p=0.049\right)$ and plasma HIV RNA $\left(r^{2}=0.089, p=0.020\right)$ were associated with NPZglobal. When both 


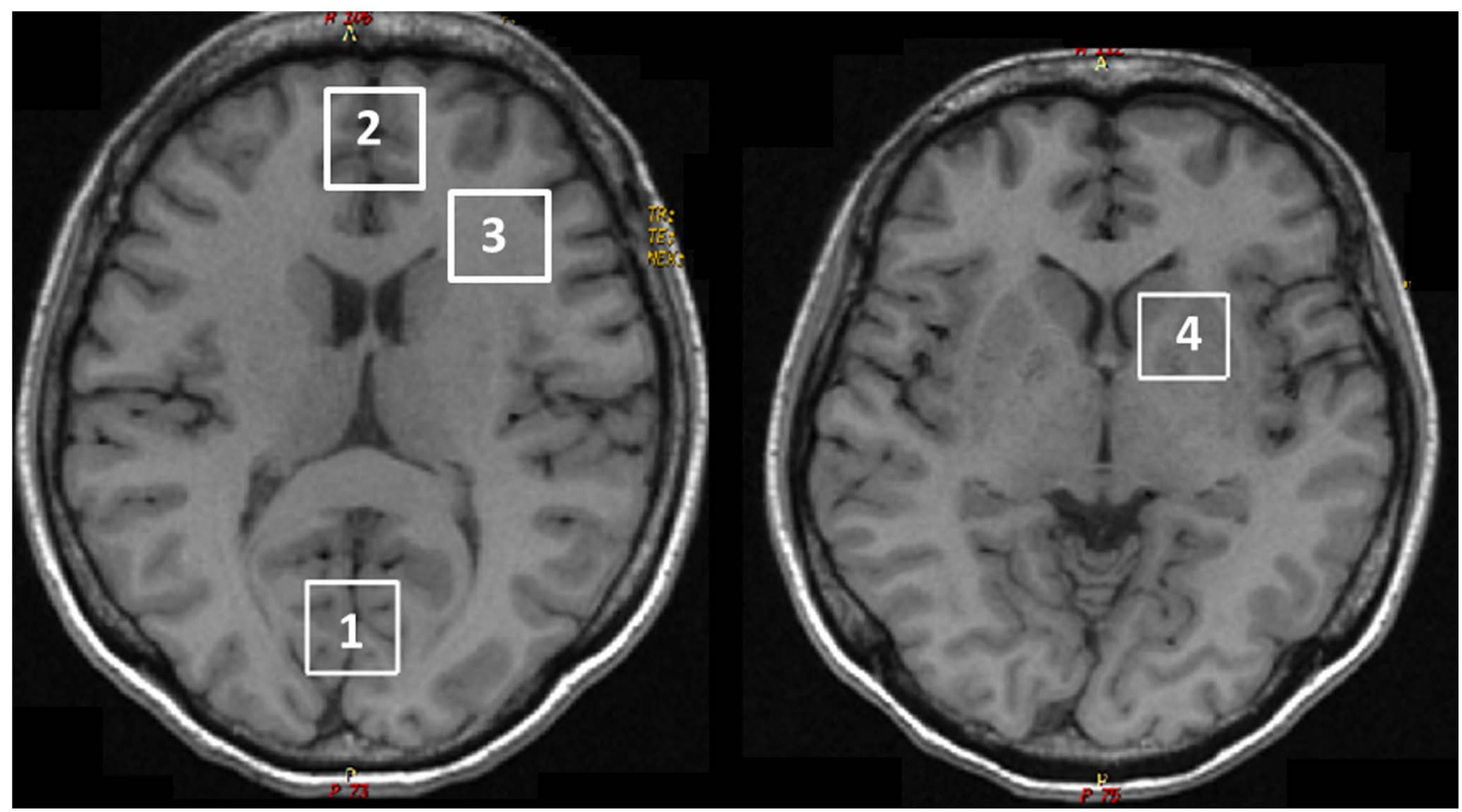

Figure 1. MRS voxel locations (representative examples). 1. Occipital grey matter, 2. Frontal grey matter, 3. Frontal white matter, 4. Basal ganglia.

doi:10.1371/journal.pone.0070164.g001

measures were included in a multiple regression model (adj. $\left.r^{2}=0.091\right)$, the effect of each was attenuated $(p=0.105$ and $p=0.041$ for HIV DNA and HIV RNA, respectively). Neither CSF HIV RNA nor CD4 ${ }^{+}$T-lymphocyte count was associated with NPZglobal. We found no association between PBMC HIV DNA and the NPZglobal.

\section{HIV DNA and MRS}

We identified an association between CD14 ${ }^{+}$HIV DNA and the two primary hypothesized metabolites, NAA and MI. The association with MI was noted in BG [point estimate (SD): 0.0224 (0.009), $p=0.0174$ ], FGM [point estimate (SD): 0.0160 (0.006), $p=0.0116$ ], and OGM [point estimate (SD): 0.015 (0.007), $p=0.033$ ] (see values in Table S1). Similarly, the association with NAA was noted in the BG [point estimate (SD): -0.01449

Table 1. Clinical characteristic of enrollees. For CSF, $n=22$ for NL and 21 for HAND groups.

\begin{tabular}{llll}
\hline & NL $(\boldsymbol{n}=33)$ & HAND $(\boldsymbol{n}=\mathbf{2 8})$ & $\boldsymbol{p}$-value \\
\hline Age, mean (SD) years & $35.3(6.5)$ & $34.0(7.4)$ & 0.373 \\
Education, mean (SD) years & $10.9(4.4)$ & $11.4(5.0)$ & 0.595 \\
Gender, $\mathrm{n}(\%)$ female & $18(54)$ & $17(60)$ & 0.627 \\
CD4 T-lymphocyte count, & $255(114,363)$ & $213(121,280)$ & 0.238 \\
median (IQR) & & & \\
$\begin{array}{l}\text { Plasma viral load, median } \log _{10} \\
\text { (SD) }\end{array}$ & $4.77(3.95,5.13)$ & $4.96(4.65,5.59)$ & 0.081 \\
CSF viral load, median $\log _{10}$ & $4.07(3.34,4.69)$ & $3.97(3.53,4.75)$ & 0.7893 \\
(SD, $n)$ & & & \\
\hline doi:10.1371/journal.pone.0070164.t001 & & & \\
\hline
\end{tabular}

(0.007), $p=0.031$ ], FGM [point estimate (SD): -0.015 (0.006), $p=0.0153$ ], and OGM [point estimate (SD): $-0.0114(0.005)$, $p=0.0260$ ] but also noted in FWM [point estimate (SD): 0.017 $(0.004), p=0.000320]$. We did not identify associations between $\mathrm{CD}_{14}{ }^{+} \mathrm{HIV}$ DNA and Cho or Glx at any voxel. Eight voxelmetabolite data points $(0.6 \%)$ were visually identified as outliers despite spectra of acceptable quality. To ensure that our findings were not driven by these outliers, we repeated the analysis excluding these data points and lost significance at the $p=0.05$ level for both MI and NAA in the basal ganglia $(p=0.09074$, $p=0.15652$, respectively). Findings in other voxels did not change appreciably.

\section{Associations to Plasma and CSF Cytokines}

Among the three cytokines of interest, HIV DNA was only associated with neopterin. This was identified in plasma $\left(r^{2}=0.066, p=0047\right)$ and CSF $\left(r^{2}=0.123, p=0.023\right)$. In models adjusted for plasma HIV RNA and $\mathrm{CD} 4^{+}$T-lymphocyte count, these associations remained significant $\left(r^{2}=0.430, p=0.014\right.$ and $r^{2}=0.328, p=0.012$ for plasma and CSF neopterin, respectively, Figure 4). Plasma IL-6 was detected in only $11 \%$ of cases, which we felt was insufficient to subject to statistical testing. HIV DNA was not associated with plasma MCP-1 $(p=0.805)$. Neither CSF IL-6 $(p=0.711)$ nor CSF MCP-1 $(p=0.679)$ were associated with HIV DNA.

\section{Discussion}

This paper summarizes the primary findings from a prospective blinded study to determine if higher intracellular HIV DNA reservoir size increases the risk for HAND and whether it is associated with CSF inflammation and MRS abnormalities. When PBMCs are purified to be enriched with monocytes $\left(\mathrm{CD} 14^{+}\right)$, we 


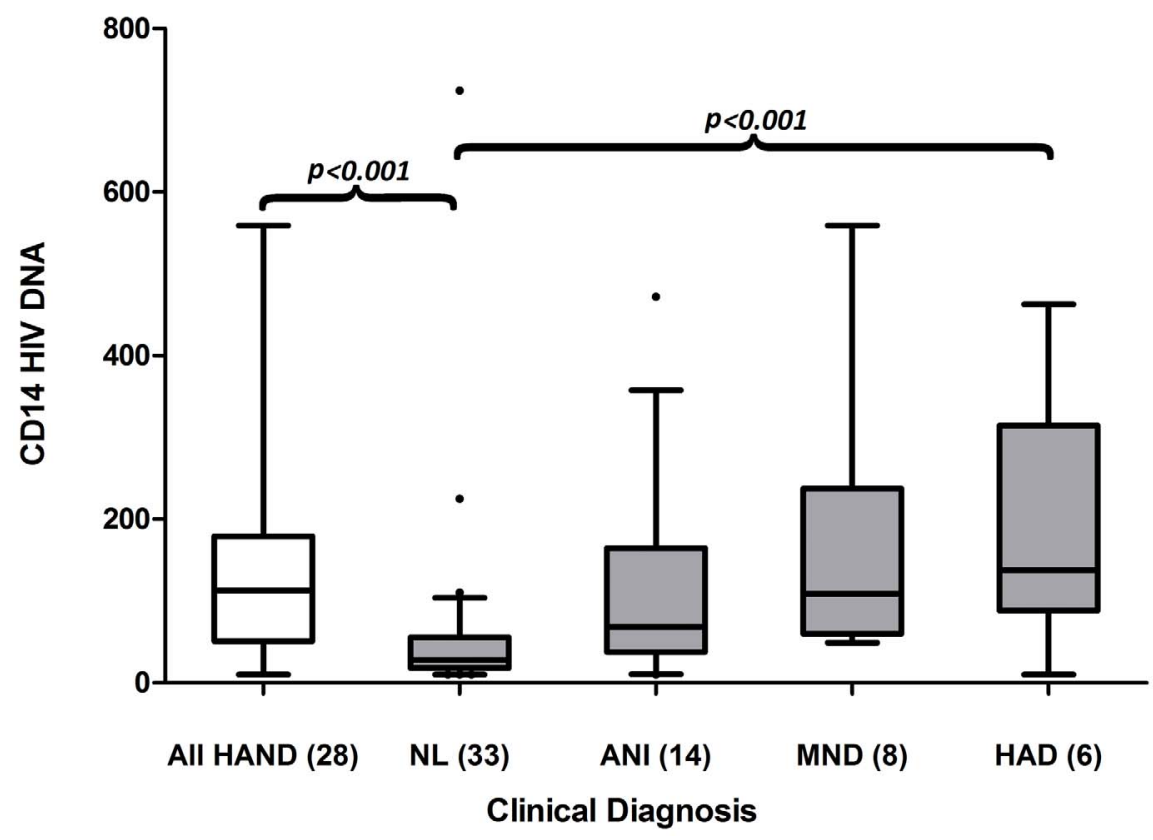

Figure 2. CD14+ HIV DNA and cognition. HIV DNA and HAND (first two bars, $p=0.0004$ ) and across diagnostic groups (last four bars, $p<0.001$ ). All $\mathrm{HAND}=\mathrm{ANI}+\mathrm{MND}+\mathrm{HAD}$.

doi:10.1371/journal.pone.0070164.g002

identified associations between the magnitude of this reservoir and HAND, poorer neuropsychological test performance, neuronal injury (reduced NAA), glial dysfunction (increased MI), and CSF immune activation (higher neopterin). Our methodology did not determine if these intracellular reservoirs are actively producing viral RNA. We designed our quantification strategy to increase the likelihood of isolating integrated viral DNA by excluding small fragments, such as those potentially forming non-integrated and non-active circular HIV DNA. Small amounts of lymphocyte contamination occurred with our $\mathrm{CD} 14^{+}$magnetic bead column

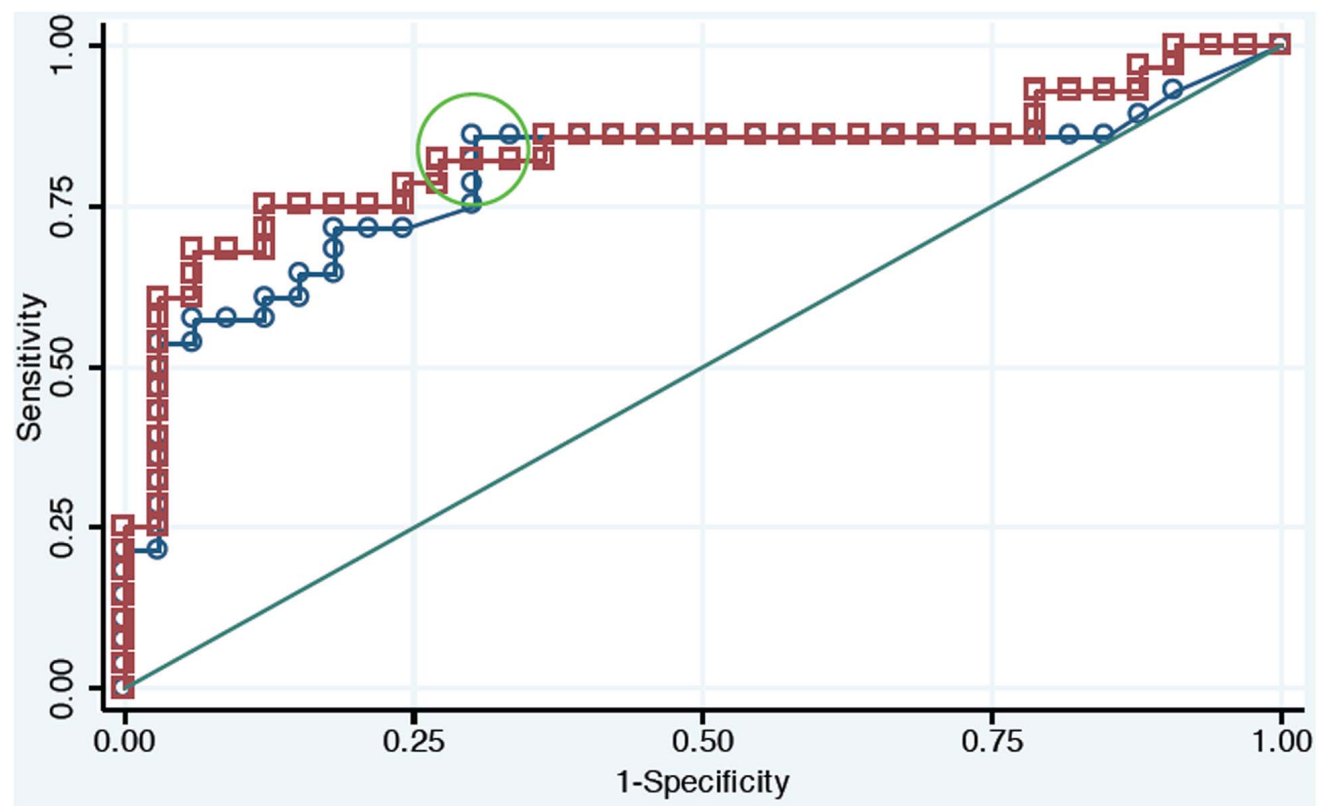

- 0 - ROC (CD14+ HIV DNA only): $\mathbf{0 . 7 9 2 2}$

—- ROC (CD14 $14^{+}$HIV DNA, CD4 $4^{+}$T-lymphocyte, and plasma HIV RNA): $\mathbf{0 . 8 2 4 7}$

Reference

Figure 3. Receiver operating characteristic (ROC) curve for level CD14 ${ }^{+}$HIV DNA identifying HAND. ROC of CD14 ${ }^{+}$HIV DNA only $=0.7922$, ROC adjusting for CD4 ${ }^{+}$T-lymphocyte and plasma HIV RNA: 0.8247.

doi:10.1371/journal.pone.0070164.g003 
Table 2. Regression analysis of the association of CD14 $4^{+}$HIV DNA and HAND.

\begin{tabular}{lll}
\hline & & \\
\hline Univariate Logistic Regression & Odds Ratio & $p$-value \\
\hline $\log _{10} \mathrm{CD} 14^{+}$HIV DNA & 8.29 & 0.002 \\
\hline Multivariate Logistic Regression & Odds Ratio & $p$-value \\
\hline $\log _{10} \mathrm{CD} 14^{+}$HIV DNA & 14.47 & 0.001 \\
$\log _{10} \mathrm{HIV}$ RNA & 2.04 & 0.167 \\
$\mathrm{CD}^{+}$T-lymphocyte count & 1.00 & 0.924 \\
\hline doi:10.1371/journal.pone.0070164.t002 & &
\end{tabular}

separation; however, the lack of association with PBMC HIV DNA increases the likelihood that the monocyte fraction drives the associations we found. Our findings suggest that the eradication of HAND may require approaches beyond standard cART since these reservoirs remain present despite treatment. [23] The impact of intracellular HIV DNA was independent of HIV RNA in plasma or CSF and of $\mathrm{CD}^{+}{ }^{+} \mathrm{T}$-lymphocyte count; however, overlapping mechanisms likely exist since the magnitude of our findings is attenuated in multivariate models. This is particularly true for the cytokine analyses, which appear to be driven in large part by HIV RNA (data not shown).

We did not identify links to HIV DNA measured in PBMCs, although these associations have been noted in previous small studies. [4,5] This is unfortunate, since isolating $\mathrm{CD}^{+} 4^{+}$cells requires added resources and time and may not be available internationally. This finding, however, is not surprising given existing theories that monocytes traffic virus to the CNS. [24] Logically, the large presence of lymphocytes in the PBMC pool would dilute the effect of monocyte HIV DNA such that associations are stronger when enriching for monocytes.

Use of biomarker evidence for CNS injury strengthens this work. As hypothesized, CD $14^{+}$HIV DNA was associated with glial dysfunction (increased MI) and neuronal injury (decreased NAA). The loss of significance in the basal ganglia when excluding outliers suggests the finding at this location was unstable and may be due to challenges in voxel shimming at this site; however no
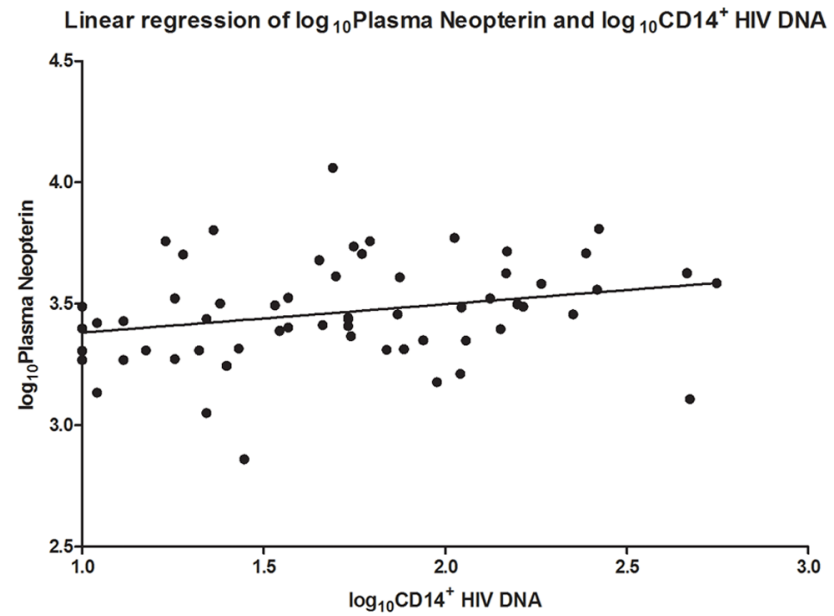

changes were noted in other regions. We did not identify associations between HIV DNA and Cho, suggestive that HIV DNA-related injury is likely due to chronic low level immune activation rather than a robust infiltrative cellular process. This is not surprising since the actual $\mathrm{CD} 14^{+}$reservoirs are small - the largest amount measured in this study was 559 copies $/ 10^{6}$ cells. We measured neopterin, MCP-1, and IL-6 due to known linkage to monocyte pathogenesis, but only neopterin was linked to HIV DNA. The lack of association between HIV DNA and MCP-1 was unanticipated, since MCP-1 has been linked to dementia. [25].

Our work extends existing paradigms by implicating intracellular reservoirs in HAND pathogenesis. In this study, all participants were naive to cART, yet we still identified a relationship that was independent of plasma or CSF viral load. These reservoirs are not universally suppressed with cART and past correlative work from our group notes associations to cognitive impairment when intracellular suppression is incomplete. $[1,5,19,26,27,28]$ This work should encourage further research into the monocyte reservoir as a therapeutic target for HAND. In a past study of treated patients with suppressed HIV RNA, a novel score of antiretroviral effectiveness in monocytes was higher in subjects without HAND. [8] Thus, the presumed concentrations of antiretrovirals in the brain, as measured by the CPE, incompletely informs the effectiveness of cART on HAND.

Another strength of this work is the availability of normative data and the use of consensus diagnostic conference for HAND. [17] The population was selected to be cART-naive but with $\mathrm{CD}^{+}$T-lymphocyte counts indicating a need to start cART ( $<350$ cells), though several cases had higher levels at enrollment. Our findings can only inform a similar population, but a longitudinal follow-up of this group is underway. The predominant HIV subtype in Bangkok is the circulating recombinant form (CRF) AE_01, but our past work notes similar characteristics of HAND in CRF AE_01 compared to other clades, and we have previously identified associations between HIV DNA and cognition in clade B infected subjects. [5,15,29] The sample size, while small, was planned to have sufficient power based on past data. Nevertheless, the effect sizes noted in comparison to the NPZglobal score appeared substantially smaller than those seen in past studies by our group. [4] These past studies enrolled participants with chronic, treated HIV infection, a suggestive finding that more profound effects may be identified in the setting

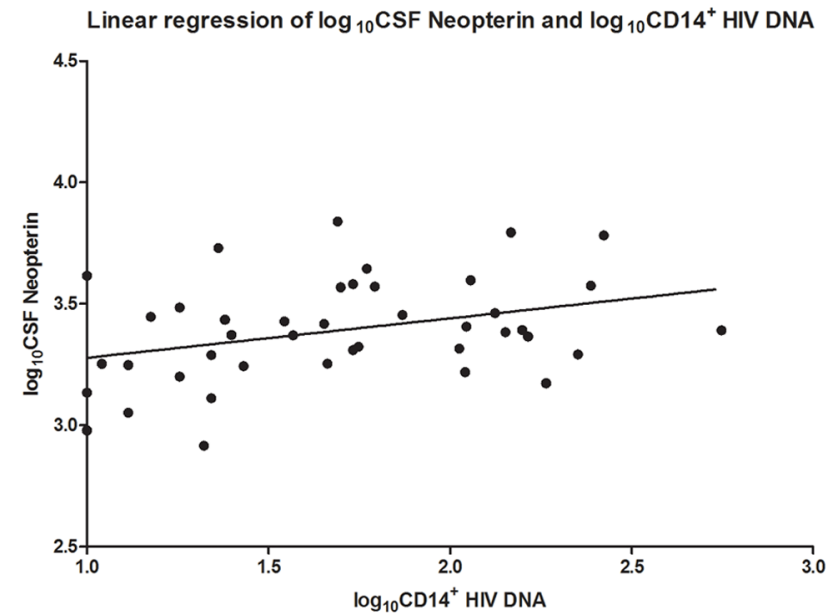

Figure 4. Plasma CD14 ${ }^{+}$HIV DNA and plasma neopterin (left, $p=0.0473$, adjusted $r^{2}=0.0662$ ) and CSF neopterin (right, $p=0.023$, adjusted $r^{2}=0.123$ ) by univariate regression.

doi:10.1371/journal.pone.0070164.g004 
of cART. It is possible that the effects of low $\mathrm{CD} 4^{+} \mathrm{T}$-lymphocyte count and high plasma HIV RNA mask the impact of HIV DNA among untreated cases.

In summary, the level of HIV DNA in circulating cells with $\mathrm{CD}_{1} 4^{+}$phenotype is related to risk for HAND in treatment-naïve Thai subjects with moderate to advanced immune suppression. This marker also correlates to MRS markers of brain injury and, nominally, to neopterin levels. New efforts are needed to understand the mechanisms by which intracellular HIV DNA levels contribute to neuronal injury.

\section{Supporting Information}

\section{Table S1 Correlation of metabolites by voxel with level of GD14+ HIV DNA. \\ (DOC)}

\section{Acknowledgments}

Membership of the SEARCH 011 protocol team: Edgar Busovaca ${ }^{1}$, Pradit Chaisit ${ }^{6}$, Yothin Chinvarun $\mathrm{MD}^{7}$, Akash Desai ${ }^{1}$, Pardis Esmaeili ${ }^{1}$, Nicholas Hutchings ${ }^{1}$, Tanate Jadwattanakul $\mathrm{MD}^{5}$, Sirichai Jarupittaya ${ }^{6}$, Prapian Khongtia ${ }^{7}$, Patrick Kirkland ${ }^{4}$, Eugeen Kroon $\mathrm{MD}^{2}$, Sukalaya

\section{References}

1. Lambotte O, Taoufik Y, de Goer MG, Wallon C, Goujard C, et al. (2000) Detection of infectious HIV in circulating monocytes from patients on prolonged highly active antiretroviral therapy. Journal of acquired immune deficiency syndromes 23: 114-119.

2. Rouzioux C, Hubert JB, Burgard M, Deveau C, Goujard C, et al. (2005) Early levels of HIV-1 DNA in peripheral blood mononuclear cells are predictive of disease progression independently of HIV-1 RNA levels and CD4+ T cell counts. J Infect Dis 192: 46-55.

3. Avettand-Fenoel V, Boufassa F, Galimand J, Meyer L, Rouzioux C (2008) HIV1 DNA for the measurement of the HIV reservoir is predictive of disease progression in seroconverters whatever the mode of result expression is. Journal of clinical virology: the official publication of the Pan American Society for Clinical Virology 42: 399-404.

4. Shiramizu B, Gartner S, Williams A, Shikuma C, Ratto-Kim S, et al. (2005) Circulating proviral HIV DNA and HIV-associated dementia. Aids 19: 45-52.

5. Shiramizu B, Paul R, Williams A, Shikuma C, Watters M, et al. (2007) HIV proviral DNA associated with decreased neuropsychological function. J Neuropsychiatry Clin Neurosci 19: 157-163.

6. Valcour VG, Shiramizu BT, Shikuma CM (2010) HIV DNA in circulating monocytes as a mechanism to dementia and other HIV complications. J Leukoc Biol 87: 621-626.

7. Valcour VG, Shiramizu BT, Sithinamsuwan P, Nidhinandana S, Ratto-Kim S, et al. (2009) HIV DNA and cognition in a Thai longitudinal HAART initiation cohort: the SEARCH 001 Cohort Study. Neurology 72: 992-998.

8. Shikuma CM, Nakamoto B, Shiramizu B, Liang CY, DeGruttola V, et al. (2012) Antiretroviral monocyte efficacy score linked to cognitive impairment in HIV. Antivir Ther 17: 1233-1242.

9. Glass JD, Fedor H, Wesselingh SL, McArthur JC (1995) Immunocytochemical quantitation of human immunodeficiency virus in the brain: correlations with dementia. Annals of neurology 38: 755-762.

10. Bell JE, Brettle RP, Chiswick A, Simmonds P (1998) HIV encephalitis, proviral load and dementia in drug users and homosexuals with AIDS. Effect of neocortical involvement. Brain : a journal of neurology 121 (Pt 11): 2043-2052.

11. Anthony IC, Ramage SN, Carnie FW, Simmonds P, Bell JE (2005) Influence of HAART on HIV-related CNS disease and neuroinflammation. Journal of neuropathology and experimental neurology 64: 529-536.

12. Ryan LA, Zheng J, Brester M, Bohac D, Hahn F, et al. (2001) Plasma levels of soluble CD14 and tumor necrosis factor-alpha type II receptor correlate with cognitive dysfunction during human immunodeficiency virus type 1 infection. J Infect Dis 184: 699-706.

13. Cantres-Rosario Y, Plaud-Valentin M, Gerena Y, Skolasky RL, Wojna V, et al. (2013) Cathepsin B and cystatin B in HIV-seropositive women are associated with infection and HIV-1-associated neurocognitive disorders. AIDS 27: 347356.
Lerdlum $\mathrm{MD}^{3}$, Pornpen Methajittiphun $\mathrm{MSc}^{5}$, Pravit Mingkwanrungruang ${ }^{6}$, Nittaya Phanuphak $\mathrm{MD} \mathrm{PhD}^{3}$, Mantana Pothisri $\mathrm{BSc}^{3}$, Somprartthana Rattanamanee $\mathrm{MSc}^{2}$, Joel Sabugo ${ }^{4}$, Pasiri Sithinamsuwan $\mathrm{MD}^{8}$, Nipat Terratakulpisarn $\mathrm{MD}^{6}$, Phayao Thongkramjaroen ${ }^{5}$, Sumit Thongmuang ${ }^{6}$, and Lauren Wendelken MS ${ }^{1}$

1 Memory and Aging Center, Department of Neurology, University of California, San Francisco, California, USA

2 SEARCH, Thai Red Cross AIDS Research Centre, Bangkok, Thailand

3 Faculty of Medicine, Chulalongkorn University, Bangkok, Thailand

4 Hawaii Center for AIDS, University of Hawaii, Honolulu, Hawaii, USA

5 Queen Savang Vadhana Memorial Hospital, Si Racha, Thailand

6 Thai Red Cross AIDS Research Centre, Bangkok, Thailand

7 Rajavithi Hospital, Bangkok, Thailand

8 Pramongkutklao Hospital, Bangkok, Thailand.

\section{Author Contributions}

Conceived and designed the experiments: VV JA NS CS DC RP MM B. Slike. Performed the experiments: JA MA NS TC AS PS ST JF MM B. Slike VD B. Shiramizu. Analyzed the data: VV JA MA NS CL DC RP MM B. Slike VD B. Shiramizu. Contributed reagents/materials/analysis tools: SJ. Wrote the paper: VV.

14. Sungkanuparph S, Techasathit W, Utaipiboon C, Chasombat S, Bhakeecheep $\mathrm{S}$, et al. (2010) Thai national guidelines for antiretroviral therapy in HIV-1 infected adults and adolescents 2010. Asian Biomedicine 4: 515-528.

15. Valcour VG, Sithinamsuwan P, Nidhinandana S, Thitivichianlert S, Ratto-Kim S, et al. (2007) Neuropsychological abnormalities in patients with dementia in CRF 01_AE HIV-1 infection. Neurology 68: 525-527.

16. Maj M, Satz P, Janssen R, Zaudig M, Starace F, et al. (1994) WHO Neuropsychiatric AIDS study, cross-sectional phase II. Neuropsychological and neurological findings. Arch Gen Psychiatry 51: 51-61.

17. Heaps JM, Valcour V, Chalermchai T, Paul R, Rattanamanee S, et al. (2012) Development of normative neuropsychological performance in Thailand for the assessment of HIV-associated neurocognitive disorders. Journal of Clinical and Experimental Neuropsychology In Press.

18. Antinori A, Arendt G, Becker JT, Brew BJ, Byrd DA, et al. (2007) Updated research nosology for HIV-associated neurocognitive disorders. Neurology 69: $1789-1799$

19. Shiramizu B, Ananworanich J, Chalermchai T, Siangphoe U, Troelstrup D, et al. (2012) Failure to clear intra-monocyte HIV infection linked to persistent neuropsychological testing impairment after first-line combined antiretroviral therapy. J Neurovirol 18: 69-73.

20. Schirmer T, Auer DP (2000) On the reliability of quantitative clinical magnetic resonance spectroscopy of the human brain. NMR Biomed 13: 28-36.

21. Wright SM, Wald LL (1997) Theory and application of array coils in MR spectroscopy. NMR Biomed 10: 394-410.

22. Provencher SW (1993) Estimation of metabolite concentrations from localized in vivo proton NMR spectra. Magn Reson Med 30: 672-679.

23. Le Douce V, Herbein G, Rohr O, Schwartz G (2010) Molecular mechanisms of HIV-1 persistence in the monocyte-macrophage lineage. Retrovirology 7: 32.

24. Gonzalez-Scarano F, Martin-Garcia J (2005) The neuropathogenesis of AIDS. Nat Rev Immunol 5: 69-81.

25. Yuan L, Qiao L, Wei F, Yin J, Liu L, et al. (2013) Cytokines in CSF correlate with HIV-associated neurocognitive disorders in the post-HAART era in China. J Neurovirol.

26. Chun TW, Stuyver L, Mizell SB, Ehler LA, Mican JA, et al. (1997) Presence of an inducible HIV-1 latent reservoir during highly active antiretroviral therapy. Proceedings of the National Academy of Sciences of the United States of America 94: 13193-13197.

27. Alexaki A, Liu Y, Wigdahl B (2008) Cellular reservoirs of HIV-1 and their role in viral persistence. Current HIV research 6: 388-400.

28. Coleman CM, Wu L (2009) HIV interactions with monocytes and dendritic cells: viral latency and reservoirs. Retrovirology 6: 51

29. Wirachsilp P, Kantakamalakul W, Foongladda S, Chuenchitra T, Kohriangudom S, et al. (2007) Surveillance of subtype and genetic variation of the circulating strains of HIV-1 in Thailand. The Southeast Asian journal of tropical medicine and public health 38: 814-827. 